\title{
Properties of an MLE algorithm for the multivariate linear model with a separable covariance matrix structure
}

\author{
Anna Szczepańska-Álvarez ${ }^{1}$, Bogna Zawieja $^{1}$, Adolfo Álvarez ${ }^{2}$ \\ ${ }^{1}$ Department of Mathematical and Statistical Methods, Poznań University of Life \\ Sciences, Wojska Polskiego 28, PL-60-637 Poznań, Poland, \\ e-mail:anna.szczepanska-alvarez@up.poznan.pl \\ e-mail: bogna.zawieja@up.poznan.pl \\ ${ }^{2}$ Collegium Da Vinci, Department of Informatics and Visual Communication, ul. \\ Kutrzeby 10, 61-719 Poznań, Poland, \\ e-mail:adolfo.alvarez-pinto@cdv.pl
}

\section{SUMMARY}

In this paper we present properties of an algorithm to determine the maximum likelihood estimators of the covariance matrix when two processes jointly affect the observations. Additionally, one process is partially modeled by a compound symmetry structure. We perform a simulation study of the properties of an iteratively determined estimator of the covariance matrix.

Key words: compound symmetry structure, Kronecker product, maximum likelihood estimation, convergence of algorithm, bias of estimator

\section{Introduction}

In experiments with high-dimensional data, one alternative to reduce the number of parameters to be estimated is the use of a model with a given covariance structure. For example, it is natural to use a model with a separable covariance structure in two-level experiments: Dutilleul and Pinel-Alloul (1996) use this model for spatio-temporal data, and the authors determine maximum likelihood estimators iteratively. Moreover, Lu and Zimmerman (2005) and Naik and Rao (2001) formulate a test to verify the separable covariance structure. Roy and Khattree (2005) and Filipiak et al. (2018) analyze the problem of testing the covariance structure, assuming additionally that one matrix has the compound symmetry structure. Manceur and Dutilleul (2013) and Nzabanita et al. (2015) consider estimation in the tensor normal model and present a simulation study concerning the properties 
of the determined estimators. Moreover, Werner et al. (2008) present two methods for obtaining estimators in models with the separable covariance structure without an iterative algorithm. Geton (2007) discusses separable approximations of space-time covariance matrices.

The assumption about the structure of the covariance matrix decreases the number of unknown parameters in the model, but the obtained estimators of these parameters usually do not have an explicit form. The estimators depend on one another and need to be determined iteratively. For example, to find the maximum likelihood estimators in a model with the separable covariance matrix structure, a two-stage algorithm has to be used. The properties of this algorithm are studied by Dutilleul (1999).

In this paper, we present the properties of iteratively determined maximum likelihood estimators in a model with a separable covariance structure where one component has a partially assumed structure. This structure can appear in an experiment where two processes jointly affect the observations. Moreover, one process is partially modeled by the compound symmetry structure. The considered covariance structure can be expected in an experiment where the characteristics are observed over time (for example, during a year) and for time points (during some season) there is the same dependence between the characteristics. We are interested in how much the assumption about the structure of the second component influences the properties of the estimators. We consider the cases where $25 \%, 50 \%$ and $75 \%$ parts of the covariance matrix have the compound symmetry structure. We study the convergence of the algorithm and the bias of estimators in this model.

The paper is organized as follows: In Section 2 we present the model and the maximum likelihood estimators, in Section 3 we provide the algorithms and the considered properties, in Section 4 we describe the simulation study and its results, and we formulate conclusions in Section 5 .

\section{Model}

Consider an experiment where $p$ characteristics are measured at $q$ time points and observations are repeated $n$ times. Moreover, the observations are independent and identically matrix normally distributed $\mathbf{X}_{i} \sim$ $N_{p, q}(\mathbf{M} ; \boldsymbol{\Psi} ; \boldsymbol{\Sigma}), \mathbf{X}_{i}: p \times q, i=1, \ldots, n$, (in vector notation: $\operatorname{vec}\left(\mathbf{X}_{i}\right) \sim$ $N_{p q}(\operatorname{vec}(\mathbf{M}) ; \boldsymbol{\Sigma} \otimes \mathbf{\Psi}), \operatorname{vec}(*)$ denotes the vec-operator from $R^{p \times q}$ to $R^{p q}$, putting columns underneath) where $\mathbf{M}$ is the mean matrix, and the matri- 
ces $\boldsymbol{\Sigma}: q \times q$ and $\boldsymbol{\Psi}: p \times p$ describe the unknown covariance matrix between the columns of $\mathbf{X}_{i}$ and between the rows of $\mathbf{X}_{i}$, respectively. Moreover, we assume that the matrix $\boldsymbol{\Sigma}$ is unstructured and the matrix $\boldsymbol{\Psi}$ has a block structure of the following form:

$$
\mathbf{\Psi}=\left(\begin{array}{cc}
\mathbf{A}(\theta) & \mathbf{B} \\
\mathbf{B}^{\prime} & \mathbf{\Omega}
\end{array}\right)
$$

where $\mathbf{A}(\theta): r \times r, 1<r<p$ is the unknown matrix which has compound symmetry structure, i.e. $\mathbf{A}(\theta)=(1-\theta) \mathbf{I}_{r}+\theta \mathbf{1}_{r} \mathbf{1}_{r}^{\prime}$, where $\mathbf{1}_{r}$ denotes the column vector of size $r$ with all elements equal to 1 , and the matrices $\mathbf{B}$ : $r \times(p-r)$ and $\boldsymbol{\Omega}:(p-r) \times(p-r)$ are unknown. In the general case, the matrices $\boldsymbol{\Sigma}$ and $\boldsymbol{\Psi}$ are not jointly identifiable, but because of the form of $\boldsymbol{\Psi}$, the matrix $\boldsymbol{\Sigma} \otimes \boldsymbol{\Psi}$ is assumed to be identifiable.

Let us write $\mathbf{Y}_{(i)}=\mathbf{X}_{i}-\hat{\mathbf{M}}, \mathbf{Y}_{(i)}: p \times q, i=1, \ldots, n$ then $\mathbf{Y}: p \times n q$, $\mathbf{Y}=\left(\mathbf{Y}_{(1)} \mathbf{Y}_{(2)} \ldots \mathbf{Y}_{(n)}\right)=\left(\begin{array}{c}\mathbf{Y}_{1} \\ \mathbf{Y}_{2}\end{array}\right)$, where $\mathbf{Y}_{1}: r \times n q, \mathbf{Y}_{2}:(p-r) \times n q$. Moreover, let $\tilde{\mathbf{Y}}: n p \times q, \widetilde{\mathbf{Y}}_{1}^{\prime}: q \times n r, \widetilde{\mathbf{Y}}_{2}^{\prime}: q \times n(p-r)$.

The maximum likelihood estimators in the above model can be written

$$
\begin{aligned}
\widehat{\theta}= & \frac{1}{n q r(r-1)} \operatorname{tr}\left(\mathbf{1}_{r} \mathbf{1}_{r}^{\prime} \mathbf{Y}_{1}\left(\mathbf{I}_{n} \otimes \widehat{\boldsymbol{\Sigma}}^{-1}\right) \mathbf{Y}_{1}^{\prime}\right)-\frac{1}{r-1}, \\
\widehat{\boldsymbol{\mu}}= & \frac{1}{n} \sum_{i=1}^{n} \mathbf{X}_{i}, \\
\mathbf{A}(\widehat{\theta})= & (1-\widehat{\theta}) \mathbf{I}_{r}+\widehat{\theta} \mathbf{1}_{r} \mathbf{1}_{r}^{\prime}, \\
n p \widehat{\boldsymbol{\Sigma}}= & \widetilde{\mathbf{Y}}_{1}^{\prime}\left(\mathbf{I}_{n} \otimes \mathbf{A}(\widehat{\theta})^{-1}\right) \widetilde{\mathbf{Y}}_{1}+\left(\widetilde{\mathbf{Y}}_{2}^{\prime}-\widetilde{\mathbf{Y}}_{1}^{\prime}\left(\mathbf{I}_{n} \otimes \widehat{\boldsymbol{\delta}}\right)\right)\left(\mathbf{I}_{n} \otimes \widehat{\mathbf{\Psi}}_{2 \bullet 1}^{-1}\right) \\
& \quad \times\left(\widetilde{\mathbf{Y}}_{2}^{\prime}-\widetilde{\mathbf{Y}}_{1}^{\prime}\left(\mathbf{I}_{n} \otimes \widehat{\boldsymbol{\delta}}\right)\right)^{\prime}, \\
\widehat{\boldsymbol{\delta}}= & \left(\mathbf{Y}_{1}\left(\mathbf{I}_{n} \otimes \widehat{\boldsymbol{\Sigma}}^{-1}\right) \mathbf{Y}_{1}^{\prime}\right)^{-1} \mathbf{Y}_{1}\left(\mathbf{I}_{n} \otimes \widehat{\boldsymbol{\Sigma}}^{-1}\right) \mathbf{Y}_{2}^{\prime},
\end{aligned}
$$

where

$$
\widehat{\mathbf{\Psi}}=\left(\begin{array}{cc}
\mathbf{A}(\widehat{\theta}) & \widehat{\mathbf{B}} \\
\widehat{\mathbf{B}}^{\prime} & \widehat{\mathbf{\Omega}}
\end{array}\right),
$$

where

$$
\begin{aligned}
\widehat{\mathbf{B}} & =\mathbf{A}(\widehat{\theta}) \widehat{\boldsymbol{\delta}}, \quad \widehat{\boldsymbol{\Omega}}=\widehat{\mathbf{\Psi}}_{\mathbf{2} \bullet \mathbf{1}}+\widehat{\boldsymbol{\delta}}^{\prime} \mathbf{A}(\widehat{\theta}) \widehat{\boldsymbol{\delta}} \\
q n \widehat{\mathbf{\Psi}}_{2 \bullet \mathbf{1}} & =\left(\mathbf{Y}_{2}-\widehat{\boldsymbol{\delta}}^{\prime} \mathbf{Y}_{1}\right)\left(\mathbf{I}_{n} \otimes \widehat{\boldsymbol{\Sigma}}^{-\mathbf{1}}\right)\left(\mathbf{Y}_{\mathbf{2}}-\widehat{\boldsymbol{\delta}}^{\prime} \mathbf{Y}_{\mathbf{1}}\right)^{\prime},
\end{aligned}
$$

(for details see Szczepańska-Alvarez et al. (2017)).

In the considered case no explicit form of the maximum likelihood estimators exists, and the above estimators can be determined iteratively. 


\title{
3. Algorithms and their properties
}

To find numerically the maximum likelihood estimators of the covariance matrix given in the previous section, we study the performance of two algorithms based on different concepts. Algorithm 1 investigates the properties of estimators determined iteratively. It studies whether the solution converges and how far it is from the assumed real matrices $\boldsymbol{\Sigma}$ and $\boldsymbol{\Psi}$. The determined estimators are based on different samples from the same distribution, but the procedure always starts from the same initial solution $\boldsymbol{\Sigma}_{\mathbf{0}}=\mathbf{I}$. Algorithm 2 searches the properties of solutions depending on the choice of the initial matrix $\boldsymbol{\Sigma}$ based on the same sample. The two algorithms can be outlined as follows:

\begin{abstract}
Algorithm 1:
step 1: generate symmetric, positive definite matrices: the unstructured $\boldsymbol{\Sigma}$ and the structured $\boldsymbol{\Psi}$ (according to the assumptions given in Section 2)

step 2: generate the sample $v e c \mathbf{Y}_{(i)}, i=1,2, \ldots, n$ from the vector normal distribution $\operatorname{vec} \mathbf{Y}_{(i)} \sim N_{p q}(\mathbf{0} ; \boldsymbol{\Sigma} \otimes \mathbf{\Psi})$, and create the matrix $\mathbf{Y}_{(i)}$

step 3: start from the initial solution $\boldsymbol{\Sigma}=\mathbf{I}_{q}$ and determine the estimators of equations 1 to 2 using the schema given by Figure 1 .

We run the algorithm 5000 times for the given sample $(l=5000)$. Moreover, we assume the maximum number of iterations to be equal to 500. If in 500 iterations we do not get a solution, we will say that the algorithm does not converge.
\end{abstract}

\section{Algorithm 2:}

step 1: generate symmetric, positive definite matrices: the unstructured $\boldsymbol{\Sigma}$ and the structured $\boldsymbol{\Psi}$ (according to the assumptions given in Section 2)

step 2: generate the sample $v e c \mathbf{Y}_{(i)}, i=1,2, \ldots, n$ from the vector normal distribution $\operatorname{vec} \mathbf{Y}_{(i)} \sim N_{p q}(\mathbf{0} ; \boldsymbol{\Sigma} \otimes \boldsymbol{\Psi})$, and create the matrix $\mathbf{Y}_{(i)}$

step 3: calculate estimators (equations 1 to 2) using the algorithm for the given sample $\mathbf{Y}_{(i)} ; i=1,2, \ldots, n$ (determined in step 2). Start from the various initial solutions from the set of positive definite matrices (see Figure 1). In this algorithm the number of starting matrices is 300 . The maximum number of iterations for the algorithm to converge is 500 .

In addition, in both algorithms we seek the convergence and bias of the solution.

We define the convergence by:

$$
\left\|\boldsymbol{\Sigma}_{t}-\boldsymbol{\Sigma}_{t-1}\right\|_{F}<\epsilon \quad \text { and } \quad\left\|\boldsymbol{\Psi}_{t}-\boldsymbol{\Psi}_{t-1}\right\|_{F}<\epsilon
$$




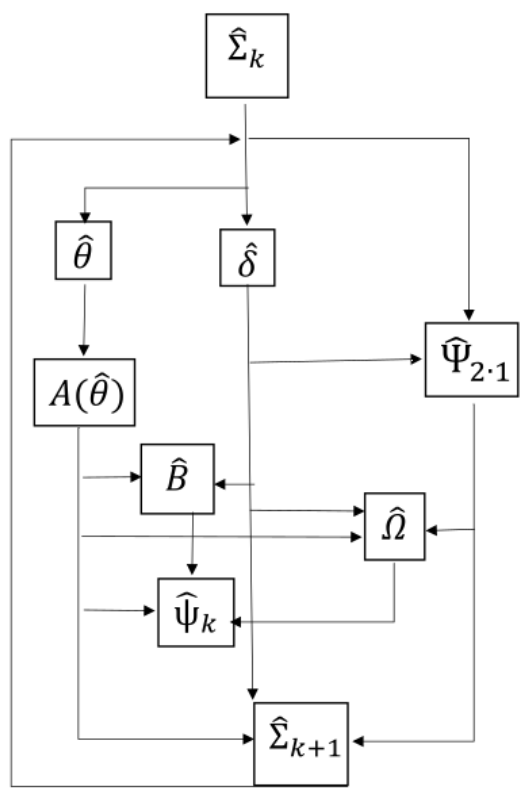

Figure 1. Schema of the algorithms

where $\epsilon=0.00001$ is the convergence criterion, $\|*\|_{F}$ means the Frobenius norm and matrices $\boldsymbol{\Sigma}_{i}, \boldsymbol{\Psi}_{i}$ are calculated in the $i$-th step. Similar criteria were used, among others, by Dutilleul and Pinel-Alloul (1996).

According to Manceur and Dutilleul (2013) and Nzabanita et al. (2015), we analyze the standardized empirical bias of the solution, determined as follows:

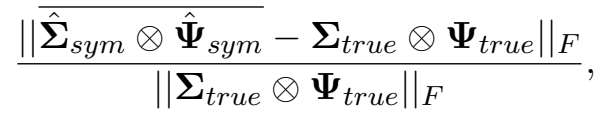

where $\overline{\hat{\boldsymbol{\Sigma}}_{\text {sym }} \otimes \hat{\mathbf{\Psi}}_{\text {sym }}}$ is the mean of the estimators obtained from the simulations, and $\boldsymbol{\Sigma}_{\text {true }}, \boldsymbol{\Psi}_{\text {true }}$ are the generated matrices assumed at the beginning of the simulations as the true covariance matrices.

Moreover, we calculate the bias for each matrix $\boldsymbol{\Sigma}$ and $\boldsymbol{\Psi}$ separable as

$$
\frac{\left\|\hat{\boldsymbol{\Sigma}}_{\text {sym }}-\boldsymbol{\Sigma}_{\text {true }}\right\|_{F}}{\left\|\boldsymbol{\Sigma}_{\text {true }}\right\|_{F}} \quad \text { and } \quad \frac{\left\|\hat{\mathbf{\Psi}}_{\text {sym }}-\boldsymbol{\Psi}_{\text {true }}\right\|_{F}}{\left\|\boldsymbol{\Psi}_{\text {true }}\right\|_{F}}
$$


where $\overline{\hat{\Sigma}}_{\text {sym }}=\frac{\sum_{j=1}^{l} \hat{\boldsymbol{\Sigma}}_{j}}{l}, \overline{\hat{\mathbf{\Psi}}}_{\text {sym }}=\frac{\sum_{j=1}^{l} \hat{\mathbf{\Psi}}_{j}}{l}$ and $l$ is the number of runs of the algorithm.

\section{Simulation study}

\subsection{Simulation parameters}

We perform a simulation study assuming the dimensions of matrices $\boldsymbol{\Sigma}: q \times q$ and $\boldsymbol{\Psi}: p \times p$ as $q=\{5,10,15\}$ and $p=\{12,24,36\}$. Moreover, we are interested in how the structure of $\boldsymbol{\Psi}$ impacts the properties of the solutions, so we consider the cases when $25 \%, 50 \%$, and $75 \%$ of the structure of the matrix $\boldsymbol{\Psi}$ is assumed. We choose $r$ as follows: $r=\{3,6,9\}$ for $p=12$, $r=\{6,12,18\}$ for $p=24, r=\{9,18,27\}$ for $p=36$. We also determine $n=\frac{q(q+1)}{2}+\frac{(p-r)(p+r+1)}{2}+2$, which is larger than the number of unknown parameters. The calculations were performed using Microsoft $\mathrm{R}$ Open 3.5.1.

\subsection{Results}

In the tables below we present the results of the simulation. First we show the properties of Algorithm 1. In Table 1 we observe the maximum, minimum and mean number of iterations when Algorithm 1 converges. The number of iterations decreases when a greater percentage of the structure of the matrix $\boldsymbol{\Psi}$ is known. Moreover, the number of simulations is more stable when $75 \%$ of the structure of $\boldsymbol{\Psi}$ is assumed. In Table 2 we get the bias for matrices $\boldsymbol{\Sigma}$ and $\boldsymbol{\Psi}$ separably. We notice that the bias is small for both matrices but slightly smaller for the matrix $\boldsymbol{\Sigma}$. Table 3 gives results for the standardized empirical bias of the solution. We observe that the bias is not large, and for given $q$, if $p$ increases the bias becomes smaller. Moreover, from both tables (Table 2 and Table 3) we observe that for fixed $q$ in each group $(25 \%, 50 \%, 75 \%)$, when the number of observations increases, the bias of both matrices $\boldsymbol{\Sigma}, \boldsymbol{\Psi}$ and the standardized empirical bias decrease. This is related to the size of $p$, which influences the sample size. Similar conclusions are obtained for Algorithm 2, but the results in that case are larger because of the smaller number of runs (see Tables 4-6). 
Table 1. The maximum, minimum and mean number of iterations in Algorithm 1

\begin{tabular}{|c|c|c|c|c|c|c|c|c|}
\hline \multirow[t]{2}{*}{$\mathrm{n}$} & \multirow[t]{2}{*}{$\mathrm{p}$} & \multirow[t]{2}{*}{$\mathrm{r}$} & \multicolumn{2}{|l|}{$25 \%$} & \multicolumn{2}{|l|}{$50 \%$} & \multicolumn{2}{|l|}{$75 \%$} \\
\hline & & & min mean & $\max$ & min mean & $\operatorname{hax}$ & min mean & $\operatorname{nax}$ \\
\hline \multicolumn{9}{|l|}{$\mathbf{q}=\mathbf{5}$} \\
\hline$\{89,74,50\}$ & 12 & $\{3,6,9\}$ & 5464.53 & 106 & 1927.40 & 48 & 1314.81 & 24 \\
\hline$\{296,239,146\}$ & 24 & $\{6,12,18\}$ & 4447.92 & 50 & 719.52 & 22 & 812.42 & 13 \\
\hline$\{638,512,305\}$ & 36 & $\{9,18,27\}$ & 4848.49 & 49 & 2323.06 & 24 & 1313.00 & 14 \\
\hline \multicolumn{9}{|l|}{$q=10$} \\
\hline$\{129,114,90\}$ & 12 & $\{3,6,9\}$ & 6572.34 & 117 & 2526.71 & 31 & 712.95 & 14 \\
\hline$\{336,279,186\}$ & 24 & $\{6,12,18\}$ & 6367.83 & 97 & 2224.29 & 31 & 611.57 & 13 \\
\hline$\{678,552,345\}$ & 36 & $\{9,18,27\}$ & 4546.78 & 48 & 2323.00 & 23 & 1314.00 & 14 \\
\hline \multicolumn{9}{|l|}{$q=15$} \\
\hline$\{194,179,155\}$ & 12 & $\{3,6,9\}$ & 6672.72 & 114 & 2425.43 & 28 & 1414.00 & 15 \\
\hline$\{401,344,251\}$ & 24 & $\{6,12,18\}$ & 1140.54 & 48 & 918.97 & 21 & 1212.41 & 13 \\
\hline$\{743,617,410\}$ & 36 & $\{9,18,27\}$ & 4848.06 & 49 & 2323.00 & 23 & 1212.83 & 13 \\
\hline
\end{tabular}

Table 2. The bias of matrices $\boldsymbol{\Sigma}$ and $\boldsymbol{\Psi}$ in Algorithm 1, where $\|\boldsymbol{\Sigma}\|,\|\boldsymbol{\Psi}\|$ are given in Eq. (5)

\begin{tabular}{|c|c|c|c|c|}
\hline \multirow[t]{2}{*}{$\mathrm{n}$} & \multirow[t]{2}{*}{$\mathrm{p}$} & $25 \%$ & $50 \%$ & $75 \%$ \\
\hline & & $\|\boldsymbol{\Sigma}\| \quad\|\boldsymbol{\Psi}\|$ & $\|\boldsymbol{\Psi}\|$ & $\|\Sigma\|$ \\
\hline \multicolumn{5}{|l|}{$\mathbf{q}=5$} \\
\hline$\{89,74,50\}$ & $12\{3,6,9\}$ & 0.00110 .0028 & 0.00100 .0023 & 0.00080 .0031 \\
\hline$\{296,239,146\}$ & $24\{6,12,18\}$ & 0.00030 .0015 & 0.00040 .0017 & 0.00050 .0021 \\
\hline$\{638,512,305\}$ & $36\{9,18,27\}$ & 0.00020 .0013 & 0.00020 .0012 & 0.00040 .0014 \\
\hline \multicolumn{5}{|l|}{$q=10$} \\
\hline$\{129,114,90\}$ & $12\{3,6,9\}$ & 0.00110 .0014 & 0.00100 .0013 & 0.00160 .0013 \\
\hline$\{336,279,186\}$ & $24\{6,12,18\}$ & 0.00050 .0011 & 0.00050 .0010 & 0.00060 .0011 \\
\hline$\{678,552,345\}$ & $36\{9,18,27\}$ & 0.00030 .0009 & 0.00030 .0008 & 0.00040 .0009 \\
\hline \multicolumn{5}{|l|}{$q=15$} \\
\hline$\{194,179,155\}$ & $12\{3,6,9\}$ & 0.00120 .0073 & 0.00130 .0006 & 0.00120 .0006 \\
\hline$\{401,344,251\}$ & $24\{6,12,18\}$ & 0.00060 .0008 & 0.00060 .0008 & 0.00080 .0008 \\
\hline$\{743,617,410\}$ & $36\{9,18,27\}$ & 0.00030 .0007 & 0.00030 .0007 & 0.00040 .0006 \\
\hline
\end{tabular}


Table 3. The standardized empirical bias (SEB) of a solution in Algorithm 1

\begin{tabular}{|c|c|c|c|c|c|}
\hline \multirow[t]{2}{*}{$\mathrm{n}$} & \multirow[t]{2}{*}{$\mathrm{p}$} & \multirow[t]{2}{*}{$\mathrm{r}$} & \multirow{2}{*}{$\frac{25 \%}{\mathrm{SEB}}$} & \multirow{2}{*}{$\frac{50 \%}{\mathrm{SEB}}$} & \multirow{2}{*}{$\begin{array}{r}75 \% \\
\text { SEB }\end{array}$} \\
\hline & & & & & \\
\hline \multicolumn{6}{|l|}{$q=5$} \\
\hline$\{89,74,50\}$ & 12 & $\{3,6,9\}$ & 0.0027 & 0.0028 & 0.0033 \\
\hline$\{296,239,146\}$ & 24 & $\{6,12,18\}$ & 0.0016 & 0.0017 & 0.0022 \\
\hline$\{638,512,305\}$ & 36 & $\{9,18,27\}$ & 0.0013 & 0.0013 & 0.0014 \\
\hline \multicolumn{6}{|l|}{$q=10$} \\
\hline$\{129,114,90\}$ & 12 & $\{3,6,9\}$ & 0.0017 & 0.0017 & 0.0023 \\
\hline$\{336,279,186\}$ & 24 & $\{6,12,18\}$ & 0.0012 & 0.0011 & 0.0009 \\
\hline$\{678,552,345\}$ & 36 & $\{9,18,27\}$ & 0.0010 & 0.0009 & 0.0010 \\
\hline \multicolumn{6}{|l|}{$q=15$} \\
\hline$\{194,179,155\}$ & 12 & $\{3,6,9\}$ & 0.0014 & 0.0015 & 0.0014 \\
\hline$\{401,344,251\}$ & 24 & $\{6,12,18\}$ & 0.0010 & 0.0009 & 0.0011 \\
\hline$\{743,617,410\}$ & 36 & $\{9,18,27\}$ & 0.0007 & 0.0007 & 0.0008 \\
\hline
\end{tabular}

Table 4. The maximum, minimum and mean number of iterations in Algorithm 2

\begin{tabular}{|c|c|c|c|c|c|c|c|c|c|}
\hline \multirow[t]{2}{*}{$\mathrm{n}$} & \multirow[t]{2}{*}{$\mathrm{p}$} & \multirow[t]{2}{*}{$\mathrm{r}$} & \multicolumn{3}{|c|}{$25 \%$} & \multicolumn{2}{|l|}{$50 \%$} & \multicolumn{2}{|l|}{$75 \%$} \\
\hline & & & $\min$ & mean & $\max$ & min mean & $\operatorname{hax}$ & min mean & \\
\hline \multicolumn{10}{|l|}{$q=5$} \\
\hline$\{89,74,50\}$ & 12 & $\{3,6,9\}$ & 73 & 101.03 & 110 & 3139.43 & 42 & 1822.21 & 24 \\
\hline$\{296,239,146\}$ & 24 & $\{6,12,18\}$ & 67 & 87.59 & 94 & 3038.08 & 40 & 1721.36 & 23 \\
\hline$\{638,512,305\}$ & 36 & $\{9,18,27\}$ & 66 & 87.53 & 94 & 3037.63 & 40 & 1720.50 & 22 \\
\hline \multicolumn{10}{|l|}{$q=10$} \\
\hline$\{129,114,90\}$ & 12 & $\{3,6,9\}$ & 72 & 98.14 & 105 & 2938.03 & 41 & 1821.84 & 23 \\
\hline$\{336,279,186\}$ & 24 & $\{6,12,18\}$ & 68 & 88.43 & 95 & 3138.74 & 41 & 1721.44 & 23 \\
\hline$\{678,552,345\}$ & 36 & $\{9,18,27\}$ & 68 & 86.53 & 92 & 3037.99 & 40 & 1720.67 & 22 \\
\hline \multicolumn{10}{|l|}{$q=15$} \\
\hline$\{194,179,155\}$ & 12 & $\{3,6,9\}$ & 71 & 96.16 & 104 & 2937.56 & 40 & 1721.51 & 23 \\
\hline$\{401,344,251\}$ & 24 & $\{6,12,18\}$ & 70 & 88.88 & 94 & 2938.85 & 41 & 1721.28 & 22 \\
\hline$\{743,617,410\}$ & 36 & $\{9,18,27\}$ & 69 & 86.37 & 92 & 3138.03 & 40 & 1721.14 & 22 \\
\hline
\end{tabular}


Table 5. The bias of matrices $\boldsymbol{\Sigma}$ and $\boldsymbol{\Psi}$ in Algorithm 2, where $\|\boldsymbol{\Sigma}\|,\|\boldsymbol{\Psi}\|$ are given in Eq. (5)

\begin{tabular}{|c|c|c|c|c|c|c|}
\hline \multirow[t]{2}{*}{$\mathrm{n}$} & \multirow[t]{2}{*}{$\mathrm{r}$} & \multicolumn{2}{|c|}{$25 \%$} & \multicolumn{2}{|c|}{$50 \%$} & $75 \%$ \\
\hline & & $\|\boldsymbol{\Sigma}\|$ & $\|\Psi\|$ & $\|\boldsymbol{\Sigma}\|$ & $\|\Psi\|$ & $\|\boldsymbol{\Sigma}\|$ \\
\hline \multicolumn{7}{|l|}{$\mathbf{q}=\mathbf{5}$} \\
\hline$\{89,74,50\}$ & $12\{3,6,9\}$ & 0.1005 & 0.1277 & 0.0800 & 0.1624 & 0.09950 .0979 \\
\hline$\{296,239,146\}$ & $24\{6,12,18\}$ & 0.0238 & 0.1103 & 0.0465 & .1079 & 0.03540 .1121 \\
\hline$\{638,512,305\}$ & $36\{9,18,27\}$ & 0.0185 & 0.0904 & 0.0263 & 0.0857 & 0.02650 .1010 \\
\hline \multicolumn{7}{|l|}{$q=10$} \\
\hline$\{129,114,90\}$ & $12\{3,6,9\}$ & 0.0989 & 0.0932 & 0.0783 & 0.0763 & 0.09590 .0785 \\
\hline$\{336,279,186\}$ & $24\{6,12,18\}$ & 0.0308 & 0.0693 & 0.0453 & 0.0664 & 0.04350 .0874 \\
\hline$\{678,552,345\}$ & $36\{9,18,27\}$ & 0.0229 & 0.0640 & 0.0212 & 0.0630 & 0.02530 .0586 \\
\hline \multicolumn{7}{|l|}{$q=15$} \\
\hline$\{194,179,155\}$ & $12\{3,6,9\}$ & 0.0661 & 0.0542 & 0.0633 & 0.0526 & 0.08580 .0510 \\
\hline$\{401,344,251\}$ & $24\{6,12,18\}$ & 0.0319 & 0.0481 & 0.0399 & 0.0477 & 0.05340 .0524 \\
\hline$\{743,617,410\}$ & $36\{9,18,27\}$ & 0.0248 & 0.0484 & 0.0260 & 0.0473 & 0.02450 .0447 \\
\hline
\end{tabular}

Table 6. The standardized empirical bias (SEB) of a solution in Algorithm 2

\begin{tabular}{|c|c|c|c|c|c|}
\hline $\mathrm{n}$ & $\mathrm{p}$ & $\mathrm{r}$ & $25 \%$ & $50 \%$ & $75 \%$ \\
\hline & & & SEB & SEB & SEB \\
\hline \multicolumn{6}{|l|}{$q=\mathbf{5}$} \\
\hline$\{89,74,50\}$ & 12 & $\{3,6,9\}$ & 0.0027 & 0.0028 & 0.0033 \\
\hline$\{296,239,146\}$ & 24 & $\{6,12,18\}$ & 0.0016 & 0.0017 & 0.0022 \\
\hline$\{638,512,305\}$ & 36 & $\{9,18,27\}$ & 0.0013 & 0.0013 & 0.0014 \\
\hline \multicolumn{6}{|l|}{$q=10$} \\
\hline$\{129,114,90\}$ & 12 & $\{3,6,9\}$ & 0.0017 & 0.0017 & 0.0023 \\
\hline$\{336,279,186\}$ & 24 & $\{6,12,18\}$ & 0.0012 & 0.0011 & 0.0009 \\
\hline$\{678,552,345\}$ & 36 & $\{9,18,27\}$ & 0.0010 & 0.0009 & 0.0010 \\
\hline \multicolumn{6}{|l|}{$q=15$} \\
\hline$\{194,179,155\}$ & 12 & $\{3,6,9\}$ & 0.0014 & 0.0015 & 0.0014 \\
\hline$\{401,344,251\}$ & 24 & $\{6,12,18\}$ & 0.0010 & 0.0009 & 0.0011 \\
\hline$\{743,617,410\}$ & 36 & $\{9,18,27\}$ & 0.0007 & 0.0007 & 0.0008 \\
\hline
\end{tabular}




\section{Conclusions}

We observe that the algorithms for the maximum likelihood estimators given by Szczepańska-Alvarez et al. (2017) converge.

The number of iterations in which the algorithm converges depends on the structure, so the larger is the percentage of the structure of the matrix $\boldsymbol{\Psi}$ that is assumed, the faster the algorithm converges. The number of iterations is quite stable, especially for the model where $75 \%$ of the structure of $\boldsymbol{\Psi}$ is assumed.

The obtained estimators in both algorithms have small bias. Moreover, it was shown in Algorithm 2 that the choice of the initial point does not influence the convergence. In all cases the algorithm converges, but for some sets of parameters $(p=36, q=\{10,15\}, r=\{9,18\}$ and $p=24, q=\{10,15\}, r=6)$ the simulations were time-consuming. These cases occurred when $25 \%$ and $50 \%$ of the structure of $\boldsymbol{\Psi}$ was assumed as compound symmetry. Moreover, for these parameters $n$ increases, and it also influences the calculation time.

\section{REFERENCES}

Dutilleul P., Pinl-Allounl B. (1996): A doubly multivariate model for statistical analysis of spatio-temporal environmental data. Environmetrics 7: 551-565.

Dutilleul P. (1999): The mle algorithm for the matrix normal distribution. Journal of Statistical Computation and Simulation 64(2): 105-123.

Filipiak K., Klein D., Mokrzycka M. (2018): Estimators comparison of separable covariance structure with one component as compound symmetry matrix. Electronic Journal of Linear Algebra 33: 83-98.

Geton M.G. (2007): Separable approximations of space-time covariance matrix. Environmetrics 18: 681-695.

Lu N., Zimmerman D.L. (2005): The likelihood ratio test for a separable covariance matrix. Statistics \& Probability Letters 73: 449-457.

Naik D.N., Rao S.S. (2001): Analysis of multivariate repeated measures data with a Kronecker product structured covariance matrix. Journal of Applied Statistics 28: $91-105$.

Nzabanita J., Von Rosen D., Singull M. (2015): Maximum likelihood estimation in the tensor normal model with structured mean. Linköping University Electronic Press.

Manceur A., Dutilleul R. (2013): Maximum likelihood estimation for the tensor normal distribution: Algorithm, minimum sample size and empirical bias and dispersion. Journal of Computational and Applied Mathematics 239(1): $37-49$. 
Roy A., Khattree R. (2005): On implementation of a test for Kronecker product covariance structure for multivariate repeated measures data. Statistical Methodology 2: 297-206.

Szczepańska-Álvarez A., Hao Ch., Liang Y., von Rosen D.(2017): Estimation equations for multivariate linear models with Kronecker structured covariance matrices. Communications in Statistics-Theory and Methods 46(16): 7902-7915.

Werner K., Jansson M., Stoica P. (2008): On estimation of covariance matrices with Kronecker product structure. IEEE Transactions on Signal Processing 56(2): 478-491. 